VII Congresso Brasileiro de Informática na Educação (CBIE 2018)

Anais do XXIX Simpósio Brasileiro de Informática na Educação (SBIE 2018)

\title{
Recomendação Baseada no Perfil e Contexto Tecnológico do Aluno
}

\author{
Paulo Rezende, Fernanda Campos, Victor Stroële, \\ Regina Braga, José Maria N. David.
}

\author{
Universidade Federal de Juiz de Fora - Programa de Pós-graduação em Ciência da \\ Computação - Rua José Lourenço Kelmer, s/n - Campus Universitário- 36036-900 Juiz \\ de Fora/MG \\ paulo.rezende, fernanda.campos, regina.braga, jose.david \\ \{@ufjf.edu.br\}; victor.stroele@ice.ufjf.br
}

\begin{abstract}
This article describes the evolution of the PERSONNA ontology for modeling the student profile and context in an e-learning environment, creating the PERSONNA-TEC ontology and its integration into the BROAD-RS architecture. This system is able to perform the recommendation of contextsensitive learning objects (LO), and enrich, flexibilize and dynamize the class through the teacher's didactic plan. The evaluation ran with students of a basic concepts computer class, with the recommendation of $L O$ for different technological devises. The results pointed to the feasibility of the proposal.
\end{abstract}

Resumo. Este artigo descreve a evolução da ontologia PERSONNA, para modelagem do perfil e do contexto do aluno em um ambiente e-learning, criando a ontologia PERSONNA-TEC e sua integração na arquitetura BROAD-RS. Esse sistema é capaz de realizar a recomendação de objetos de aprendizagem (OA) sensível ao contexto, e enriquecer, flexibilizar e dinamizar os conteúdos de uma disciplina através do plano didático do professor. A avaliação ocorreu com alunos da disciplina de Conceitos Básicos do Computador com recomendações de OA para recursos tecnológicos diferenciados. Os resultados obtidos apontam para a viabilidade da proposta.

\section{Introdução}

$\mathrm{Na}$ área educacional os sistemas de recomendação (SR) extraem e utilizam informações sobre o contexto e perfil dos alunos, dentre elas suas preferências, objetivos, estilos de aprendizagem e dados dos dispositivos tecnológicos utilizados. Conhecer essas características permite recomendar recursos educacionais de forma individual e personalizada. Estas informações devem estar padronizadas a fim de evitar inconsistências semânticas e facilitar a interoperabilidade [HINZ, 2011]. Um modelo de contexto e perfil que privilegie a semântica das informações e a padronização dos conceitos, pode contribuir para o reuso de recursos educacionais. Neste sentido, o contexto é o ambiente físico ou conjunto de circunstâncias (como o local e o tempo) a partir do qual se considera um fato que ajuda a compreender os recursos educacionais envolvidos. Já o perfil está relacionado às caracterísiticas específicas da pessoa a ser recomendado o recurso educacional. Assim, um modelo que captura de maneira adequada tanto o perfil quanto o contexto do usuário, permitindo sua evolução ao longo do tempo e com a possibilidade de descoberta de informações implícitas relacionadas ao usuário é de grande importância.

A ontologia PERSONNA [REZENDE et al., 2015] é um modelo conceitual, que reune informações sobre perfil educacional, incluindo regras lógicas relacionadas, com vistas a ser utilizada em sistemas de recomendação ou outras aplicações educacionais. A ontologia foi utilizada em trabalhos anteriores do Projeto BROAD [Pereira et al. 2018] [Pereira et al. 2014a] [Pereira et al. 2014b] [Pereira et al. 2015] [Almeida et al. 2016] 
VII Congresso Brasileiro de Informática na Educação (CBIE 2018)

Anais do XXIX Simpósio Brasileiro de Informática na Educação (SBIE 2018)

para inferir recomendações de objetos de aprendizagem (OA) de acordo com o contexto e perfil dos alunos. O contexto tecnológico foi inicialmente descrito de forma superficial e genérica, com a expansão da classe DEVICE. Neste trabalho, apresentamos uma evolução dos trabalhos anteriores, com o objetivo de expandir a ontologia PERSONNA, a partir da especificação da ontologia PERSONNA-TEC, incluindo então um detalhamento contextual relacionado a dispositivos tecnológicos. Assim, a ontologia PERSONNA-TEC, contextualiza as informações dos recursos utilizados pelos alunos e foi utilizada no contexto da arquitetura BROAD-RS para verificar sua adequabilidade na recomendação de recursos educacionais, considerando o contexto tecnológico.

A arquitetura BROAD-RS (BROAD Recommendation System) [Rezende et al. 2013] é capaz de apoiar a recomendação de OA sensível ao contexto, e assim enriquecer, flexibilizar e dinamizar os conteúdos de uma disciplina. Uma outra característica dessa arquitetura é a possibilidade do professor selecionar os objetos de aprendizagem que comporão o plano didático. A questão que se coloca como pesquisa é: integrando a PERSONNA-TEC na arquitetura BROAD-RS, esse sistema é capaz de identificar o contexto tecnológico dos alunos para recomendar os OA do plano didático do professor aderentes aos diferentes dispositivos usados pelos alunos? Neste sentido, este artigo apresenta as extensões realizadas e um estudo de caso onde o uso da ontologia, juntamente com o BROAD-RS é avaliada, em um ambiente real de uma disciplina básica de Ciência da Computação.

O artigo está organizado da seguinte forma: na seção 2 são apresentados os trabalhos relacionados. Na seção 3 é apresentada a arquitetura BROAD-RS e uma breve descrição da ontologia PERSONNA-TEC e detalhadas subclasses. Na seção 4 está a avaliação da proposta e, por fim, a seção 5 apresenta as considerações finais.

\section{Trabalhos Relacionados}

Os modelos e propostas de sistemas de recomendação de recursos educacionais podem ser analisados pelos tipos de abordagens, tecnologias, recursos, métodos $\mathrm{e}$ características adotados. As ontologias representam uma tecnologia importante para representação de perfil e contexto. Carvalho (2016) apresenta uma ontologia para apoio a recomendações que consideram os estilos de aprendizagem, não focando em contexto. Entretanto, a ontologia proposta por Dolog e Nejdl (2007) e o modelo proposto por Verbert et al. (2012) foram considerados os mais adequados para serem utilizados como base para a criação da ontologia PERSONNA por representarem modelos de perfil e contexto. Em termos de arquiteturas que utilizam as ontologias em sistemas de recomendação destacamos a proposta de Ferreira (2017) que apresenta uma abordagem baseada em ontologias para a modelagem e avaliação do estudante em SAIEs (Sistemas Adaptativos e Inteligentes para Educação). A principal função desse modelo é acompanhar as atividades desenvolvidas pelo estudante e determinar um nível de desempenho com base no seu conhecimento e comportamento. Melo (2017) propõe um modelo do estudante baseado em emoções e perfis de personalidade com intuito de desenvolver o perfil afetivo do estudante em Ambientes Virtuais de Aprendizagem. Nascimento (2016) descreve uma experiência de mobile learning, a partir da percepção docente em relação ao desenvolvimento de um projeto pedagógico.

Assim a ontologia PERSONNA-TEC vem para expandir a ontologia PERSONNA apliando sua base de modelagem do perfil e contexto do aluno, automatizando o processo de aquisição das informações com maior precisão. 
VII Congresso Brasileiro de Informática na Educação (CBIE 2018)

Anais do XXIX Simpósio Brasileiro de Informática na Educação (SBIE 2018)

\section{BROAD-RS: Sistema para Recomendação de OA Sensível ao Contexto}

A arquitetura BROAD-RS [Rezende et al., 2015] tem o objetivo de realizar recomendações dos OA aderentes aos interesses e necessidades do aluno com o uso de uma base semântica e sistema de agentes. A abordagem deste trabalho considera a avaliação pelo professor dos OA para compor o plano didático, definindo-se a sequência e a ordem de recomendação dos recursos, considerando as tarefas, objetivos, competências e o conteúdo educacional da disciplina.

A base semântica da arquitetura é composta pela ontologia PERSONNA [Rezende et al., 2015], complementada pela ontologia PERSONNA-TEC, para modelagem do perfil e contexto do aluno além de ontologias de domínio de áreas de conhecimento e de metadados de OA. O detalhamento dos contextos tecnológicos complementa a base semântica inicialmente proposta. O plano didático na arquitetura BROAD-RS permite o professor selecionar e avaliar os OA que serão considerados para a recomendação. Através dessa avaliação, a relevância desses objetos para o aluno pode ser identificada, e então, uma recomendação personalizada pode ser gerada.

A BROAD-RS utiliza filtragem baseada em conteúdos e é sensível ao contexto dos usuários. As informações de contexto consideradas são: dimensões externas (ambiente e localização) e dimensões internas (usuário, social, preferências, atividades, objetivos e competências). São características do ambiente: a localização do aluno, as suas características, hora do dia, estado do ambiente físico, estado do sistema computacional, histórico de interações do estudante, entre outras.

A arquitetura está estruturada em quatro níveis, conforme apresentado na Figura 1: Backend Layer - camada que armazena as ontologis e os OA. É nesta camada também que a aplicação armazena os planos didáticos e as recomendações já realizadas; Middle Layer - camada de serviços, utilizada para acessar as aplicações da arquitetura. É responsável pela interação dos agentes com a base de conhecimento. É nesta camada que se encontram serviços de criação, gravação e recuperação de dados de contexto, perfil e plano didático, e serviços relacionados ao processamento das inferências nas ontologias; Multi-agent Layer - camada que descreve todos os agentes, seus respectivos papeis e suas inter-relações e interações com as aplicações. Os agentes relacionam os dados de contexto, perfil do aluno e plano didático do professor com a rede de ontologias; Client Layer - implementa a interface e telas dos usuários que podem ser desenvolvidas e integradas em qualquer Learning Management System (LMS), como um plugin da Ambiente Virtual de Aprendizagem Moodle.

Os agentes da arquitetura BROAD-RS são baseados na reatividade (por reagir a estímulos/mensagens recebidas de outros agentes) com habilidade de socializar, pois verificam as interações dos professores e alunos no sistema, como atualização de dados de contexto e plano didático. Os agentes também verificam a interação dos dados com as ontologias e serviços da arquitetura para realizar recomendações de OA de maneira mais dinâmica e ágil.

A implementação do Middle Layer foi desenvolvida em linguagem JAVA, utilizando as tecnologias JADE para agentes e JENA para lidar com as ontologias em OWL. O Reasoner utilizado foi o Pellet e OWLAPI e comandos SPARQL para criar consultas semânticas nas ontologias. Foram utilizadas também tecnologias como Hibernate, Framework ZK Boss e banco de dados MySQL. Outras ferramentas de desenvolvimento foram o Protégé, o NetBeans como IDE da linguagem JAVA e Phpmyadmin para a estruturação do banco de dados relacional. Para capturar os dados dos dispositivos foi utilizado o software CPD-Z. A escolha deste utilitário foi baseada em vários fatores, tais como: quantidade de recursos e configurações disponveis da 
VII Congresso Brasileiro de Informática na Educação (CBIE 2018)

Anais do XXIX Simpósio Brasileiro de Informática na Educação (SBIE 2018)

ferramenta; compatibilidade com a maioria dos sistemas operacionais existentes e compatível com os tipos de dispositivos conhecidos.

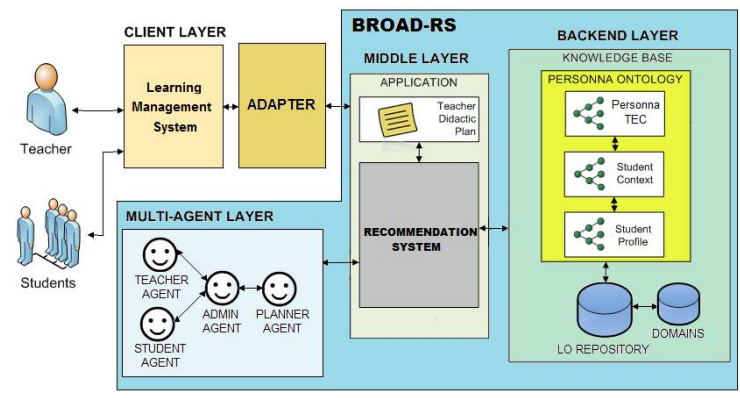

Figura 1 - Arquitetura BROAD-RS

\subsection{PERSONNA-TEC: uma ontologia para contexto e perfil do aluno}

A ontologia PERSONNA [Rezende et al., 2015] tem por objetivo ser a base semântica para a modelagem do perfil e contexto do aluno em um ambiente e-learning, a fim de padronizar as informações e facilitar o processo de recomendação de objetos de aprendizagem. A Figura 2 apresenta uma visão da ontologia, mostrando suas principais classes e subclasses que incorporam os atributos mais relevantes dos alunos em relação aos modelos e padrões existentes.

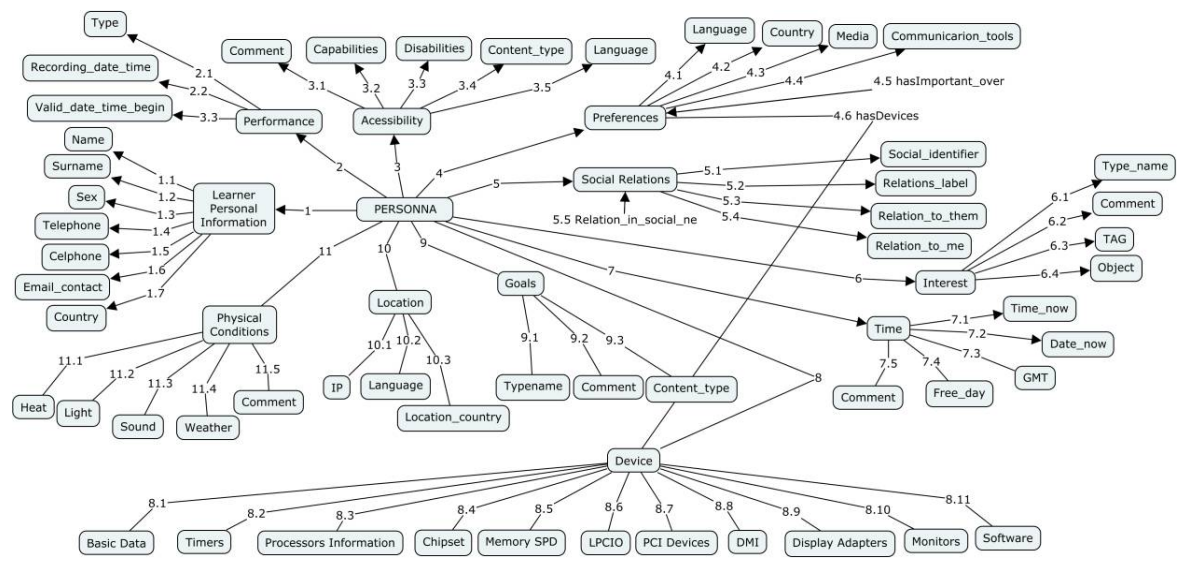

Figura 2: Visão da Ontologia PERSONNA com classes e subclasses

A Ontologia PERSONNA-TEC vem para expandir a ontologia PERSONNA e visa padronizar as informações tecnológicas facilitando o processo de recomendação de objetos de aprendizagem aderentes aos dispositivos utilizados pelo aluno. A classe "DEVICE" (Figura 3) representa esses dispositivos.

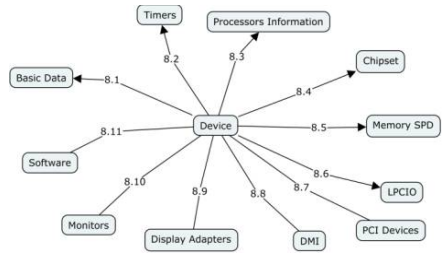

Figura 3: Visão Geral da Classe Device

Para esta pesquisa foram considerados 18 dispositivos de 4 categorias: 5 smartfones, 4 tablets, 6 computadores desktop e 4 notebooks. Algumas características são particulares para cada tipo de dispositivo, por exemplo, dispositivos móveis geralmente têm sensores de posição. Assim, foi realizada uma análise destes metadados considerando os mais frequentes, essenciais e de melhor importância em vários tipos de dispositivos. A seguir descrevemos algumas classes: 
VII Congresso Brasileiro de Informática na Educação (CBIE 2018)

Anais do XXIX Simpósio Brasileiro de Informática na Educação (SBIE 2018)

Classe Basic Data: A classe Basic_Data armazena informações básicas do dispositivo do usuário, tais como: tamanho, peso, tipo, descrição, fabricante, modelo, numero de série e data de fabricação.

Classe Display Adapters: Na classeDisplay_Adapters, ilustrada na Figura 4, estão as informações sobre os dispositivos gráficos do equipamento, como o modelo da placa de vídeo, marca, tipo de tecnologia, processador gráfico GPU (Graphics Processing Unit), memória dedicada, entre outras informações. O limite de resolução do dispositivo são fundamentais para contextualizar o equipamento do usuário e recomendar conteúdo educacional compatível, como: vídeos, jogos, livros, entre outros.

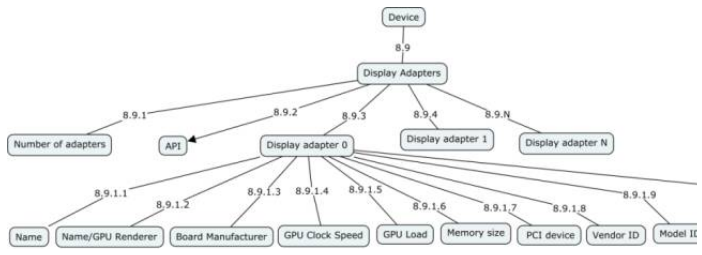

Figura 4: Classe: Device - Display Adapters

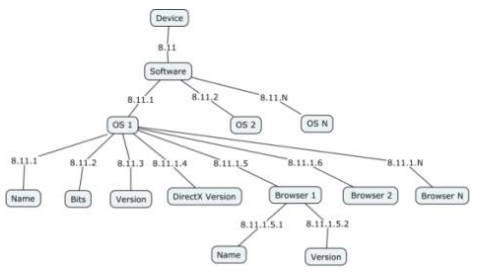

Figura 5: Classe: Device - Software

Classe Software: Na classe Software, ilustrada na Figura 5, tem-se informações de software, como a versão completa do sistema operacional, service_pack (pacote de distribuição), suporte ao formato de arquivo específico e versões de bibliotecas DirectX e Browsers instalados no dispositivo.

Classe Monitors: Na classe Monitors estão informações sobre as saídas de vídeo do dispositivo como telas LCD, projetores ou monitores. É possível identificar a resolução máxima do equipamento para a execução de um vídeo e para adaptação e acessibilidade do aluno.

\section{Avaliação em Contextos Tecnológicos Diferenciados}

A avaliação do sistema de recomendação do BROAD-RS, com uso da ontologia PERSONNA-TEC é descritiva, feita através de um Estudo de Caso [Drescht et al. 2015]. No contexto educacional um estudo de caso exige a descrição completa de uma atividade, com diferentes situações iniciadas e finalizadas através de eventos, resultado de ações do aluno sobre o ambiente educacional ou vindas do próprio ambiente. Para responder a questão de pesquisa inicialmente descrita foi feita uma demonstração da operação prática do SR, utilizado em um ambiente real de aprendizagem, sensível à situação de aprendizagem do estudante por parte do ambiente. Mostrou-se também quais elementos contextuais devem ser analisados a cada momento e as possíveis recomendações a serem realizadas.

A avaliação ocorreu na disciplina de Conceitos Básicos do Computador para alunos dos cursos da área de Computação (Ciência da Computação, Sistemas de Informação e Licenciatura em Computação), da Universidade Federal de Juiz de Fora. Nesta ocasião, foram realizadas recomendações dos objetos de aprendizagem préselecionados pelo professor para alunos que utilizam recursos tecnológicos diferenciados. A avaliação seguiu as seguintes etapas: definição, formulação do objetivo, planejamento, execução/observação e apresentação dos resultados. Após o cadastro, seleção e avaliação de objetos de aprendizagem pelo professor da disciplina, e a elaboração um plano didático de um tópico da disciplina, foi então realizada a recomendação desses objetos compatíveis com o perfil e contexto dos alunos. O repositório estava disponível com 60 OA cadastrados no padrão de metadados.

Foram utilizados os seguintes grupos de elementos contextuais: dados pessoais e comportamentais (nome, sexo, cursos matriculados, disciplina, línguas conhecidas (fluência), conhecimento, preferências no uso do ambiente educacional, e forma de navegação no conteúdo didático); dados do domínio (dados relativos aos OA disponíveis ao aluno (domínio educacional) e modelo educacional criado pelo professor); dados tecnológicos (dados a respeito do dispositivo computacional utilizado para navegação no conteúdo didático); dados de localização (local onde o aluno está situado); e dados de tempo (tempo corrente de acesso ao sistema educacional). 
VII Congresso Brasileiro de Informática na Educação (CBIE 2018)

Anais do XXIX Simpósio Brasileiro de Informática na Educação (SBIE 2018)

\subsection{Estudo de Caso}

O Estudo de Caso teve como objetivo demonstrar a viabilidade da infraestrutura BROAD-RS, integrada a um Ambiente Virtual de Aprendizagem (AVA) com a utilização da PERSONNA-TEC, em relação à recomendação de objetos de apredizagem sensíveis ao perfil e contexto e aderentes aos diferentes recursos tecnológicos utilizados pelos alunos. O cenário educacional inclui uma disciplina, os objetos de aprendizagem e o modelo didático e os atores, que são o professor e estudantes. O quadro 1 descreve as etapas executadas para a avaliação.

\begin{tabular}{|c|c|c|c|c|}
\hline Etapa & Ator & Descrição & Passos & BROAD-RS \\
\hline 1 & $\begin{array}{c}\text { Suporte } \\
\text { Administrador }\end{array}$ & $\begin{array}{c}\text { Adição da } \\
\text { infraestrutura BROAD- } \\
\text { RS ao Moodle } \\
\end{array}$ & $\begin{array}{l}\text { 1. Adicionar a } \\
\text { infraestrutura BROAD-RS } \\
\text { ao AVA }\end{array}$ & - Integração com o AVA \\
\hline II & $\begin{array}{l}\text { Suporte } \\
\text { Professor }\end{array}$ & $\begin{array}{l}\text { Inclusão de OA no } \\
\text { repositório de } \\
\text { conteúdos } \\
\text { educacionais } \\
\end{array}$ & $\begin{array}{l}\text { 2. Acessar a disciplina } \\
\text { 3. Adicionar recursos } \\
\text { 4. Selecionar permissões } \\
\text { 5. Descrever conteúdo } \\
\end{array}$ & $\begin{array}{l}\text { - Cadastro de OA de acordo com os } \\
\text { metadados }\end{array}$ \\
\hline III & Professor & $\begin{array}{c}\text { Avaliação e Seleção } \\
\text { de OA }\end{array}$ & $\begin{array}{l}\text { 6. Definir o plano didático } \\
\text { da disciplina }\end{array}$ & $\begin{array}{l}\text { - Registro do plano do professor } \\
\text { - Uso do Agente TeacherAgent }\end{array}$ \\
\hline IV & Estudante & $\begin{array}{l}\text { Alteração de perfil e } \\
\text { contexto }\end{array}$ & $\begin{array}{l}\text { 7. Acessar o AVA } \\
\text { 8. Alterar dispositivo, } \\
\text { localização, entre outros }\end{array}$ & $\begin{array}{l}\text {-Verificação de mudança de } \\
\text { contexto } \\
\text { - Uso das ontologias } \\
\text { - Uso do Agente StudentAgent } \\
\text { - Execução da máquina de inferên- } \\
\text { cia } \\
\end{array}$ \\
\hline V & & Acesso à disciplina & $\begin{array}{l}\text { 9. Verificar OA } \\
\text { recomendados }\end{array}$ & $\begin{array}{l}\text { - Uso do Agente PlannerAgent } \\
\text { - Uso das ontologias } \\
\text { - Realização de recomendação de } \\
\text { OA aderentes ao contexto e perfil }\end{array}$ \\
\hline
\end{tabular}

A execução do estudo de caso ocorreu em diferentes contextos, tendo como personagem a Estudante A, aluna do Curso de Graduação em Ciência da Computação, da UFJF. Os dados foram coletados numa quinta-feira do mês de março de 2018, quando a estudante acessou o Moodle e começou a usar o sistema de recomendação BROAD-RS em seus dispositivos tecnológicos. Nessa ocasião, a Estudante A tinha um Smartfone, um Tablet, um Notebook e um PC em sua residência e estava cursando as disciplinas básicas de introdução ao curso. As características dos dispositivos tecnológicos utilizados pela aluna, estão descritos baseados no modelo ontológico da PERSONNA-TEC (Quadro 2).

\begin{tabular}{|c|c|c|c|c|}
\hline & Smartfone & Tablet & Notebook & PC \\
\hline \multicolumn{5}{|c|}{ Classe Processors Informations } \\
\hline Processamento & Baixo & Baixo & Médio & Alto \\
\hline \multicolumn{5}{|c|}{ Classe Memory SPD } \\
\hline Memória & Baixo & Baixo & Alto & Alto \\
\hline \multicolumn{5}{|c|}{ Classe Display Adapters } \\
\hline Resolução & Média & Média & Alto & Alto \\
\hline Máxima Obtida & SD 480 & HD 720 & FULLHD 1080 & UHD 4K \\
\hline \multicolumn{5}{|c|}{ Classe Monitors } \\
\hline Tela/Tamanho (Polegadas) & 4,5 & 7 & 17 & 22 \\
\hline É TouchScreen? & Sim & Sim & Sim & Não \\
\hline \multicolumn{5}{|c|}{ Classe Basic Data } \\
\hline Mobilidade & Alta & Alta & Média & Nenhuma \\
\hline \multicolumn{5}{|c|}{ Classe Software } \\
\hline Sistema Operacional & Android & Android & Windows & Linux e Windows \\
\hline Navegador & Chrome & Chrome & Firefox & Firefox \\
\hline Suporte ao Flash? & Não & Não & Sim & Sim \\
\hline Suporte ao DirectX? & Não & Não & Sim & Sim \\
\hline Suporte Javascript? & Sim & Sim & Sim & Sim \\
\hline \multicolumn{5}{|c|}{ Outras Classes } \\
\hline Tipo de Rede & 3G/WiFi & $3 \mathrm{G} / \mathrm{WiFi}$ & 3G/WiFi/Cabo & WiFi/Cabo \\
\hline Possui impressora? & Não & Não & Não & Sim \\
\hline Possui saida de Som? & Sim & Não & Sim & Sim \\
\hline
\end{tabular}

Quadro 2 - Classes identificadas da PERSONNA-TEC

Na Etapa I, o Suporte/Administrador acessou o Moodle, fez o upload do 
VII Congresso Brasileiro de Informática na Educação (CBIE 2018)

Anais do XXIX Simpósio Brasileiro de Informática na Educação (SBIE 2018)

pluginmod-broad-rs a partir de um arquivo compactado e o instalou (Figura 6).

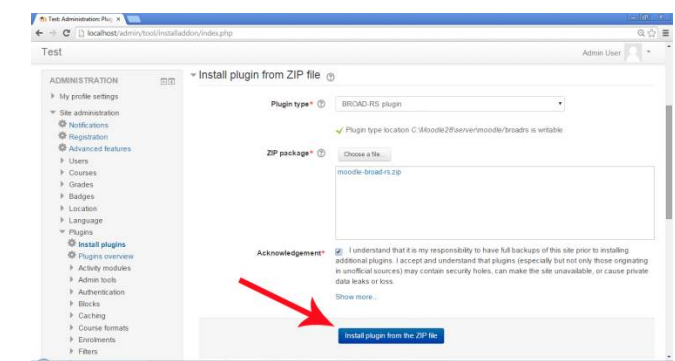

Figura 6 - Etapa I - Instalação do Plugin BROAD-RS no Moodle

Na Etapa II foi feita a inclusão de OA no repositório pelo professor preenchendo os metadados (Figura 7). Na Etapa III, o professor definiu o plano didático avaliou e selecionou os OA recomendados para os alunos (Figura 8).

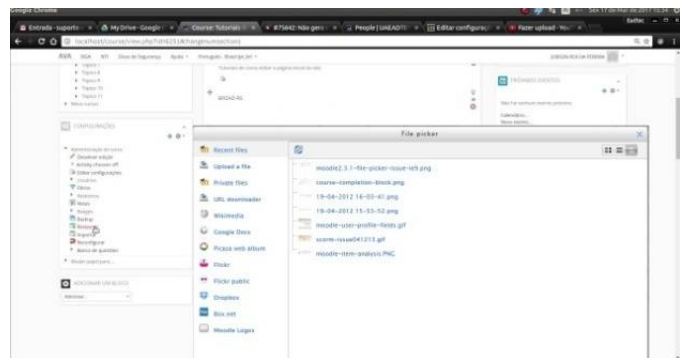

Figura 7 - Etapa II - Inclusão de OA no repositório pelo professor

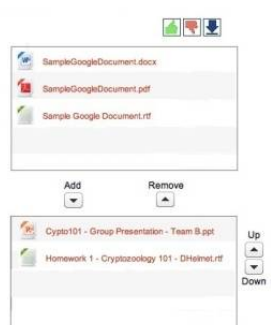

Figura 8 - Etapa III - O professor I define os OA do plano didático

As Etapas IV e $\mathrm{V}$ foram realizadas várias vezes tendo em vista os diferentes acessos da estudante ao Moodle ao longo do dia, permitindo a atuação do BROAD-RS em diferentes contextos. No primeiro acesso, as 9:30h, indo para universidade, a Estudante A usou seu Smartfone conectado a uma rede 3G. As condições são: sinal baixo de internet e tela pequena e pouco processamento. O sistema recomendou um OA com tamanho abaixo de 40kb, vídeos de curta duração com resolução baixa condicionada a tela. A Estudante A abriu um arquivo de PDF com o título: Unidade I (Figura 9).

No restaurante universitário, as 11:30h, a Estudante A usou seu tablet conectado a uma rede Wifi. As condições foram: sinal médio/alto de internet e tela média e pouco processamento. O sistema BROAD-RS recomendou um OA com tamanho até 500MB e vídeos no máximo $\mathrm{HD}$. A Estudante A recebeu um link para um video enviado pelo seu professor sobre Conceitos Básicos do Computador (Figura 10).

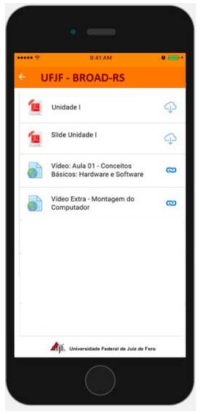

Figura 9 - Altera contexto Smartphone

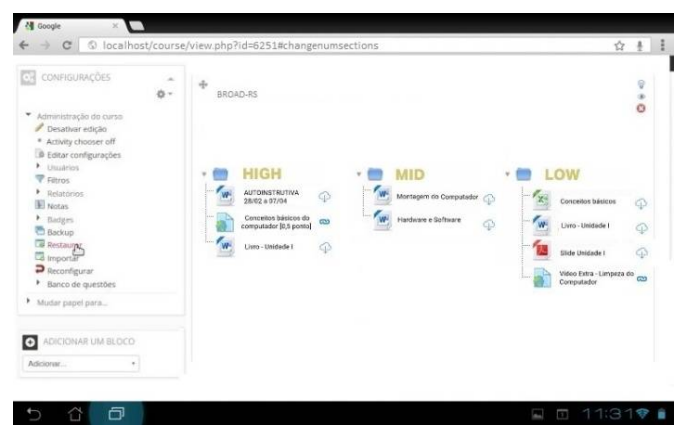

Figura 10 -Altera perfil e contexto - Tablet

Na biblioteca, as 12:30, a Estudante A usou seu tablet conectado a uma rede 3G. As condições foram: sinal baixo de internet e tela média e pouco processamento $\mathrm{O}$ sistema BROAD-RS recomendou um OA com tamanho abaixo de 100kb, vídeos de 
VII Congresso Brasileiro de Informática na Educação (CBIE 2018)

Anais do XXIX Simpósio Brasileiro de Informática na Educação (SBIE 2018)

curta duração com resolução baixa condicionados a tela. A Estudante A abriu um documento sobre montagem do computador e o leu rapidamente por ser curto.

Na cantina, as 13:30h, a Estudante A usou seu smartfone e verificou que não tinha 3G ou rede Wifi disponível. As condições foram: nenhum sinal de internet. A Estudante A reviu o arquivo, no formato PDF, sobre a aula passada, denominado Slide Unidade I já previamente carregado. Ainda na cantina, a estudante A usou seu notebook conectado a uma rede $3 \mathrm{G}$ usando acesso à internet móvel. As condições foram: sinal fraco de internet. O sistema BROAD-RS recomendou um OA com tamanho abaixo de $40 \mathrm{~kb}$, e vídeos de curta duração. A Estudante A recebeu o link de uma imagem disponibilizada em um servidor denominada "AUTOINSTRUTIVA 28/02 a 07/04". Tratava-se de uma imagem de um folheto do congresso comentado pelo seu professor, um arquivo pequeno e com boa resolução (condicionado ao tamanho do monitor de seu notebook) (Figura 11).

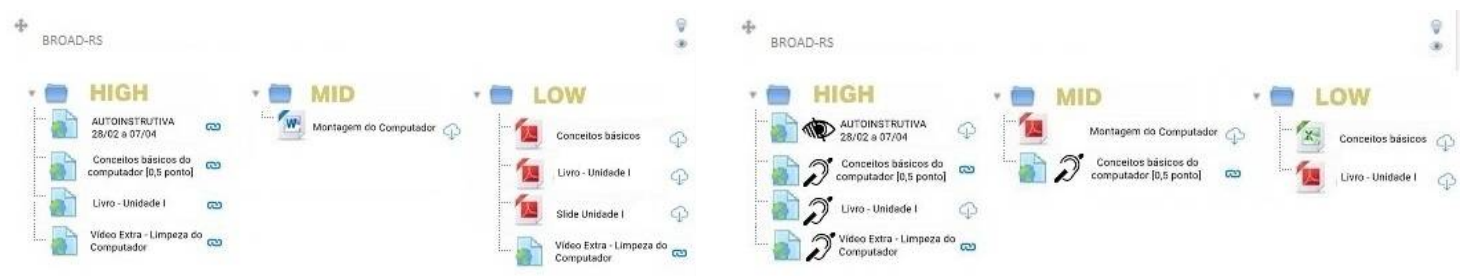

Figura 11 -Altera perfil e contexto Notebook

Figura 12 - Altera perfil e contexto Notebook

Na Biblioteca, as 14:33h, a Estudante A usou seu notebook conectado a uma rede wifi. A Estudante A emprestou seu notebook para seu amigo, Estudante B, que tem baixa audição. O contexto tecnológico não foi alterado, mas o agente UserAgent verificou a mudança de usuário, assim, recomendou somente $\mathrm{OA}$ condicionados à sua necessidade, no caso: vídeos com Libras. O sistema BROAD-RS recomendou OA sem áudio. Em seguida, o Estudante B devolveu o notebook para a Estudante A (Figura 12).

Na sala de aula, as 15:50h, a Estudante A usou seu notebook conectado a uma rede wifi. O sistema BROAD-RS, ao final da aula, recomendou, automaticamente, o material da aula, selecionado previamente pelo professor, considerando dados de contexto como o tempo e localização dos alunos (Figura 13).

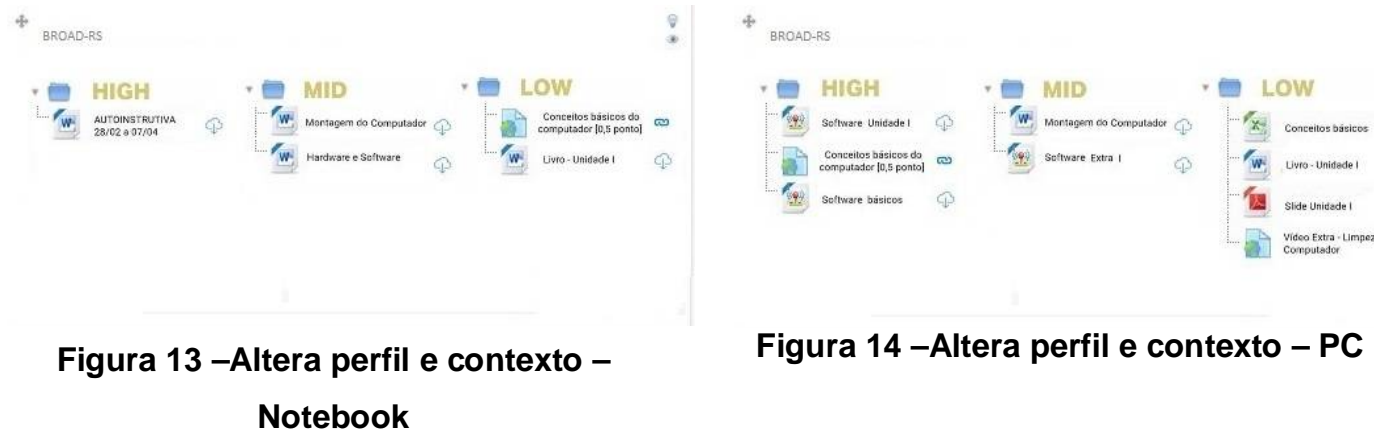

Em casa, a noite, as 18:50h, a Estudante A usou seu PC conectado a uma rede cabeada banda larga. As condições foram: sinal ótimo de internet. Logo após, o sistema BROAD-RS recomendou um OA com tamanho livre e vídeos. Foi verificado que o sistema recomendou alguns materiais, mas priorizou um jogo interativo condicionado à velocidade de processamento do dispositivo e às características da sua placa de vídeo 3D (Figura 14).

\subsection{Evidências Observadas}


VII Congresso Brasileiro de Informática na Educação (CBIE 2018)

Anais do XXIX Simpósio Brasileiro de Informática na Educação (SBIE 2018)

O Estudo de Caso demonstrou a viabilidade da proposta da arquitetura BROAD-RS, dos conceitos e das tecnologias envolvidas, do uso das ontologias PERSONNA e PERSONNA-TEC, na integração de sistema de recomendação no AVA, cobrindo um ciclo completo de uso da mesma no Moodle. Observou-se que o detalhamento da PERSONNA-TEC inclui muitos itens que podem ser usados apenas nas super-classes. Foi possível também observar que, do ponto de vista de atores que não possuiam conhecimentos técnicos, como Professor e Estudantes, o uso do BROAD-RS não exigiu habilidades técnicas específicas. Na perspectiva do Professor, a adição de objetos de aprendizagem e seus metadados no repositório exigiu conhecimento prévio sobre os mesmos, notadamente sobre itens relativos ao uso educacional dos mesmos. Em termos dos itens recomendados os mesmos foram considerados aderentes pela estudante em suas avaliações. Foi comprovada a utilização de contextos computacionais diferenciados com o uso de vários equipamentos e a necessidade de serem consideradas essas características nas recomendações. Por questões de espaço não foi possível descrever a aplicação do plano didático para mais alunos, com perfis e contextos diferentes.

\section{Considerações Finais}

A sensibilidade ao contexto vêm ganhando espaço no desenvolvimento de sistemas e aplicações que passam a apresentar ênfase na análise do contexto dos usuários e das situações configuradas no decorrer do tempo, buscando fazer com que os sistemas possam, proativamente, perceber as melhores condições de ação e agir de acordo com esta percepção.

Nesse artigo foi apresentada a evolução da ontologia, agora denominada PERSONNA-TEC. Através da análise do software CPU-Z, foi possível verificar características comuns em diversos dispositivos que compõem os termos da proposta. Um estudo de caso de uma situação de aprendizagem real foi apresentado.

Essa pesquisa pode contribuir para ampliar a utilização de objetos de aprendizagem através de uma sistematização do processo de recomendação de conteúdos educacionais aderentes a um plano do professor e características dos alunos, sensíveis ao tempo e aos dispositivos tecnológicos. A experiência obtida com o desenvolvimento deste trabalho nos levou a concluir que o desenvolvimento de ambientes sensíveis ao contexto tende a exigir o desenvolvimento de OA mais ricos, pois precisam prever diferentes situações de aprendizagem e buscar meios mais adequados para apresentação do conteúdo educacional. Estamos trabalhando num modelo de Ecossistema para Sistemas de recomendação que irá auxiliar no desenvolvimento desses aplicativos na área educacional.

\section{Agradecimentos}

À FAPEMIG, CNPq, UFJF e CAPES pelo apoio financeiro ao projeto. Aos professores e alunos da UFJF que participaram da avaliação da proposta.

\section{Referências}

Almeida, R., Pereira, C. K., Campos, F. e Ströele, V. (2016). Recomendação de recursos educacionais para grupos: buscando soluções em redes sociais. In Anais do XXVII Simpósio Brasileiro de Informática na Educação - SBIE 2016, v 27, p 966.

Carvalho, V. C.; Mendes, M. M., Ferreira, H. N. M.; Dorça, F. A.; Cattelan, R. G. (2016) Uma Ontologia para Apoio à Recomendação Automática e Personalizada de Conteúdo Considerando Estilos de Aprendizagem de Estudantes em Sistemas Adaptativos para Educação. . In Brazilian Symposium on Computers in Education Simposio Brasileiro de Informática na Educação - SBIE 2016, v 27, p 1175.

Dolog, P.; Nejdl, W. (2007) Semantic Web Technologies for the Adaptive Web. The 
VII Congresso Brasileiro de Informática na Educação (CBIE 2018)

Anais do XXIX Simpósio Brasileiro de Informática na Educação (SBIE 2018)

Adaptive Web, Springer-Verlag, Berlin, Heidelberg, p. 697-719.

Drescht, A.; Lacerda, D.P.; Antunes Junior, J.A.V. (2015). Design Science Research: método para avanço da ciência e tecnologia. Porto Alegre, Bookman, p 204.

Ferreira, H. N. M. ; Araújo, R. D.; Dorça, F. A., Cattelan, R. G. (2017) Uma Abordagem Baseada em Ontologias para Modelagem e Avaliação do Estudante em Sistemas Adaptativos e Inteligentes para Educação. Anais do XXVIII Simpósio Brasileiro de Informática na Educação - SBIE 2017, v 28, p 1197.

Hinz, V. T.; Gasparini, I.; Pimenta, M. S. (2011) Em busca de recomendação de objetos de aprendizagem em um ambiente e-learning com uso de ontologias - In: LACLO 2011 - Sexto Congresso Latinoamericano de Objetos de Aprendizagem, Montevidéu. LACLO 2011 - Sexto Congresso Latinoamericano de Objetos de Aprendizagem.

IEEE. LOM - Learning Object Metadata Working Group 12. (2012). Disponível em: http://www.ieeeltsc.org:8080/Plone/working-group/ Acesso em: 7 de maio de 2018.

Melo, S. L.; Dantas, A. C.; Fernandes, M. A. (2017) Modelo do estudante baseado em emoçõoes e perfis de personalidade para recomendação de estratégias pedagógicas personalizadas. Anais do XXVIII Simpósio Brasileiro de Informática na Educação SBIE 2017, v 28, p 967.

Nascimento, K. A. S., Filho, J. A. C. (2016) Dispositivos móveis na educação: ensinando e aprendendo em diferentes contextos. Anais do XXVII Simpósio Brasileiro de Informática na Educação - SBIE 2016, v 27, p 1225.

Pereira, C. K., Campos, F., Ströele, V., David, J. M. N. and Braga, R. (2018). BROADRSI - educational recommender system using social networks interactions and linked data. Journal of Internet Services and Applications, v. 9, n. 1, p 7.

Pereira, C. K.; Campos, F. C. A.; David, J. M. N.; Braga, R. M. (2014). Extracting User Profile and Context from Social Networks and Virtual Learning Environments to Recommend Educational Resources, ICWI, Porto, Portugal: [s.n.], p.171-178.

Pereira, C. K.; Campos, F.; Ströele, V. (2014) Extração de Características de Perfil e de Contexto em Redes Sociais para Recomendação de Recursos Educacionais. In: Simpósio Brasileiro de Informática na Educação, 2014, Dourados-MS. Anais do SBIE 2014 p 506-515. Porto Alegre: SBC.

Pereira, C.K., Campos, F., Ströele, V., N, J.M. and Braga, R. (2015). Explorando Dados Ligados através de um Sistema de Recomendação Educacional. Anais do XXVI Simpósio Brasileiro de Informática na Educação - SBIE 2015, v 26, p. 1042.

Rezende, P., Campos, F., Braga, R. and David, J. M. N. (2013). Broad-rs: uma arquitetura para recomendação de objetos de aprendizagem sensível ao contexto usando agentes. Anais do X Congresso Brasileiro de Ensino Superior a Distância, p. 11-13.

Rezende, P.; Campos, F.; David, J. M. N.; Braga, R. (2015) PERSONNA: proposta de ontologia de contexto e perfil de aluno para recomendação de objetos de aprendizagem, RBIE (Revista Brasileira de Informática na Educação) V.23 N.1 2015, janeiro.

Verbert, K.; Manouselis, N.; Ochoa, X. (2012) Context-Aware Recommender Systems for Learning: A Survey and Future Challenges, IEEE Transactions on Learning Technologies, v. 99, no. PrePrints. 